\title{
Spatiotemporal boundaries of linear vection
}

\author{
XAVIER M. SAUVAN \\ University Hospital, Zurich, Switzerland \\ and \\ CLAUDE BONNET \\ Université Louis Pasteur, Strasbourg, France
}

\begin{abstract}
Thresholds for the perception of linear vection were measured. These thresholds allowed us to define the spatiotemporal contrast surface sensitivity and the spatiotemporal domain of the perception of rectilinear vection (a visually induced self-motion in a straight line). Moreover, a Weber's law was found, such that a mean relative differential threshold in angular velocity of about $41 \%$ is necessary to perceive curvilinear vection. This visually induced self-motion corresponds to the sensation of moving in a curved path. It is proposed that curvilinear vection is induced when the apparent velocity difference is detectable. The spatiotemporal domain of perception of rectilinear vection and its spatiotemporal contrast surface sensitivity are centered on low spatial frequencies. Concurrently, the values which correspond to the relative differential thresholds of curvilinear vection are low spatial frequencies. Accordingly, the peripheral ambient visual system seems to be involved in perceiving linear vection. It is argued further that the central ambient system might also be involved in the processing of linear vection.
\end{abstract}

In this experimental work, we are concerned with the study of thresholds for the perception of linear vection. It is one part of a more general work on linear vection, the other part being the study of the properties of curvilinear vection (Sauvan \& Bonnet, 1988, 1989, 1993).

Vections are exocentric motions which consist of the perception of an apparent self-motion in an unmoving environment. Vections can be triggered by a moving visual stimulus, or by vestibular and visual stimuli (Dichgans \& Brandt, 1978). The perceived direction of the induced selfmotion is always opposite to the direction of the stimulation (Berthoz \& Droulez, 1982; Dichgans \& Brandt, 1978). The visual and the vestibular systems work in a complementary and overlapping way. Visual and vestibular inputs converge in the vestibular nuclei (Waespe \& Henn, 1977, 1978), the visual input via a route involving the nucleus of the optic tract and the nucleus reticularis tegmenti pontis (Howard, 1986), at the thalamic level (Büttner \& Henn, 1976; Büttner \& Lang, 1979) and at the cortical level (Büttner \& Buettner, 1978; Büttner \& Lang, 1979; Sauvan \& Peterhans, 1995; Vanni-Mercier \& Mag-

The experiments described in this article were carried out in the Laboratory of Experimental Psychology (UA 316 CNRS and Universite René Descartes, Paris). We thank J. Rauschecker and R. van der Zwan for helpful comments on versions of the manuscript. We are grateful to $\mathrm{N}$. Bonora and C. Bogey for their technical assistance, and to G. Loakes for improving the English. Finally, we thank Myron Braunstein for helpful suggestions on the manuscript. This research was supported by Grant ESPRIT 6615 Mucom II to X.M.S. Correspondence concerning this article should be addressed to X. M. Sauvan, Department of Neurology, University Hospital, Frauenklinikstrasse 26, CH-8091 Zurich, Switzerland. nin, 1982). Finally, a dominance of the visual system in processing visuovestibular information can be observed in the case of circular vection (Büttner \& Henn, 1981; Probst, Straube, \& Bles, 1985), or in the case of rectilinear vection (Berthoz \& Droulez, 1982; Berthoz, Pavard \& Young, 1975). This result may be related not only to the existence of neurons in the dorsal part of the medial superior temporal visual area (MSTd) in the monkey which strongly respond to optical flow stimuli (Lappe \& Rauschecker, 1993; Tanaka \& Saito, 1989; Wurtz \& Duffy, 1992) but also to the centrifugal organization of neuronal direction preferences found in the extrastriate visual area PMLS in the cat (Rauschecker, von Grünau, \& Poulin, 1987). ${ }^{1}$

Most of the experimental research on vection is related to circular vection - that is, an induced rotation about the vertical axis. Few experimental studies refer not only to sagittal linear vections (rectilinear or curvilinear vection) but also to their spatiotemporal domain of perception. Rectilinear vection is a visually induced self-motion in a straight line, forward or backward (Berthoz et al., 1975). Curvilinear vection corresponds to the sensation of taking a bend (Sauvan \& Bonnet, 1989, 1993), and it contains rotational and translational components (Andersen, 1986). It is similar to actual observer movement in a curved path, as when one is moving within a turning vehicle.

It is possible to induce vection by stimulating only a part of the visual field, such as the central visual field, for example. Indeed, a rectilinear vection is perceived when a radially expanding dot pattern subtending $7.5^{\circ}$ of visual angle is the inducing stimulus (Andersen \& Braunstein, 1985). Moreover, circular vection can be induced by stimulating only the central visual field, and not the peripheral field (Post, 1988). These results do not support the pe- 
ripheral dominance hypothesis (Brandt, Dichgans, \& Koenig, 1973).

Optical flow has also been shown to affect postural stability (see, e.g., Lestienne, Soechting, \& Berthoz, 1977). Stoffregen (1985) did not find that peripheral stimulation of the retina alone is the most efficient to induce body sway, but he did show that the stimulated retinal region (central vs. peripheral) and the optical flow structure (radial vs. lamellar ${ }^{2}$ ) is involved in postural stability. Indeed, there is a complete lack of compensatory body sway elicited by even the largest exposure of radial optical flow to the retinal periphery (Stoffregen, 1985). Concurrently, lamellar optical flow in the visual periphery is effective in inducing compensatory body sways (Stoffregen, 1985). Correspondingly, it was suggested that differentiations between areas of the optic array are also based on variations in flow structure in children (Stoffregen, Schmuckler, \& Gibson, 1987). However, Stoffregen (1985) could not estimate the actual limit of the central retina's ability to pick up visual information in affecting postural stability. It has been shown that postural adjustments occur with central visual stimulation subtending $15^{\circ}$ of visual angle-that is, when this stimulation is restricted to a small area of the central visual field (Andersen \& Dyre, 1989). This suggests that optical information from the central visual field is used to determine spatial orientation and that peripheral stimulation is not necessary to affect postural stability (Andersen \& Dyre, 1989). Postural adjustments were sampled at a higher sampling rate in Andersen and Dyre's (1989) study than in Stoffregen's (1985) work. Consequently, their measurement system may have been more sensitive to the low amplitude of sway produced by visual stimulation of the central visual field.

In the present study, visual stimulation was used to induce rectilinear vection. Vection can also be induced, for example, by using both visual and vestibular stimulations (Dichgans \& Brandt, 1978). It has been shown that central rectilinear ${ }^{3}$ (Andersen \& Braunstein, 1985), or horizontal linear ${ }^{4}$ (Telford, Spratley, \& Frost, 1992) vection is influenced by depth cues - that is, by complex visual information. For example, apparent depth, introduced by using kinetic occlusion information, influences the strength of horizontal linear vection (Telford et al., 1992). Concurrently, radial displays 5 in which there are internal depth cues, such as changing optical velocity and size, induce linear vection (Telford \& Frost, 1993). These internal depth cues are sufficient for triggering linear vection with radial displays (Telford \& Frost, 1993). Moreover, when no internal depth cues are available, as in lamellar displays ${ }^{6}$ the depth segregation required to induce linear self-motion can be given by monocular occlusion information (Telford \& Frost, 1993). These results reinforce the proposal that the higher level ambient system, which may process visual information such as depth cues (cf. Andersen \& Braunstein, 1985), is involved in the perception of complex induced self-motion (Sauvan \& Bonnet, 1993). This we define as observer's (or robot) motions along linear and/or curved paths, especially in a cluttered environment.
We measured thresholds for perceiving linear vection in order to determine the specific limitations involved in the computation of (linear) self-motion. Very few studies have examined thresholds for linear vection. It has been shown that the low velocity threshold for inducing rectilinear vection is close to that for detecting the movement of a peripheral image, and that the luminance threshold for perceiving rectilinear vection is also close to that for detecting peripheral image motion (Berthoz et al., 1975).

Thresholds for perceiving rectilinear vection and curvilinear vection are measured in Experiments 1 and 3, respectively. Thresholds for perceiving rectilinear vection and detecting the movement of drifting sine wave gratings are compared in Experiment 2.

It was found that the thresholds for perceiving linear vection define a spatiotemporal domain of perception for linear vection centered on the low spatial and the middle temporal frequencies, with the thresholds for detecting sine wave gratings movement lying outside of that spatiotemporal zone. Curvilinear vection was generated in the present experimental work by means of peripheral stimulation of low spatial frequency, but covering only a small area. Perceived velocity and degree of curvature of curvilinear vection vary differently (Sauvan \& Bonnet, 1993). Moreover, we have shown that perceived direction of curvilinear vection is not always reported unambiguously (Sauvan \& Bonnet, 1989). These results suggest that the lower and the higher level ambient systems, according to Andersen and Braunstein's (1985) theory, should be involved in the perception of linear vection.

\section{GENERAL METHOD}

\section{Subjects}

Five young adults - 3 emmetropic females (M.B., N.B., and C.T.), 1 emmetropic male (J.P.R.), and 1 myopic male (X.S.), between the ages of 22 and 28 , took part in at least one experiment. X.S. was the only subject who knew the purposes of the experiments.

\section{Apparatus}

Each subject sat with his/her head on a chinrest and was directed to gaze at the fixation point (Figure 1).

Two Tektronix monitors 604 (P31) were displayed behind two circular apertures. Each aperture subtended a visual angle of $23^{\circ}$. The monitors stimulated corresponding retinal points. The distance between the subject's eyes and the screens of the monitors was $25 \mathrm{~cm}$. Leibowitz, Rodemer, and Dichgans (1979) have shown that vection is independent of the refractive error. Consequently, the small distance between the monitors and the subject's eyes should not have had an effect on the perceived vection. These apertures were set up in a half-sphere located in a dark cabin. The mean luminance of the two monitors was $4.15 \mathrm{~cd} / \mathrm{m}^{2}$. The centers of the screens of these monitors were placed at an eccentricity of $60^{\circ}$ on either side of the subject's sagittal plane. This experimental situation produced separate stimulation of the two eyes (dichoptic vision). The stimuli were vertical sine wave gratings moving in the nasotemporal direction and generated on the screens of the monitors by a Picasso CRT image generator under computer control (Z80 microcomputer system). The contrast of the gratings is defined as $\left(L_{\max }-L_{\min }\right) /\left(L_{\max }+L_{\min }\right)$, in which $L_{\max }$ and $L_{\min }$ are the maximum and minimum luminances of the gratings, respectively. The subjects and image generator were located in two different rooms to reduce the noise in the subjects' room as much as possible. 


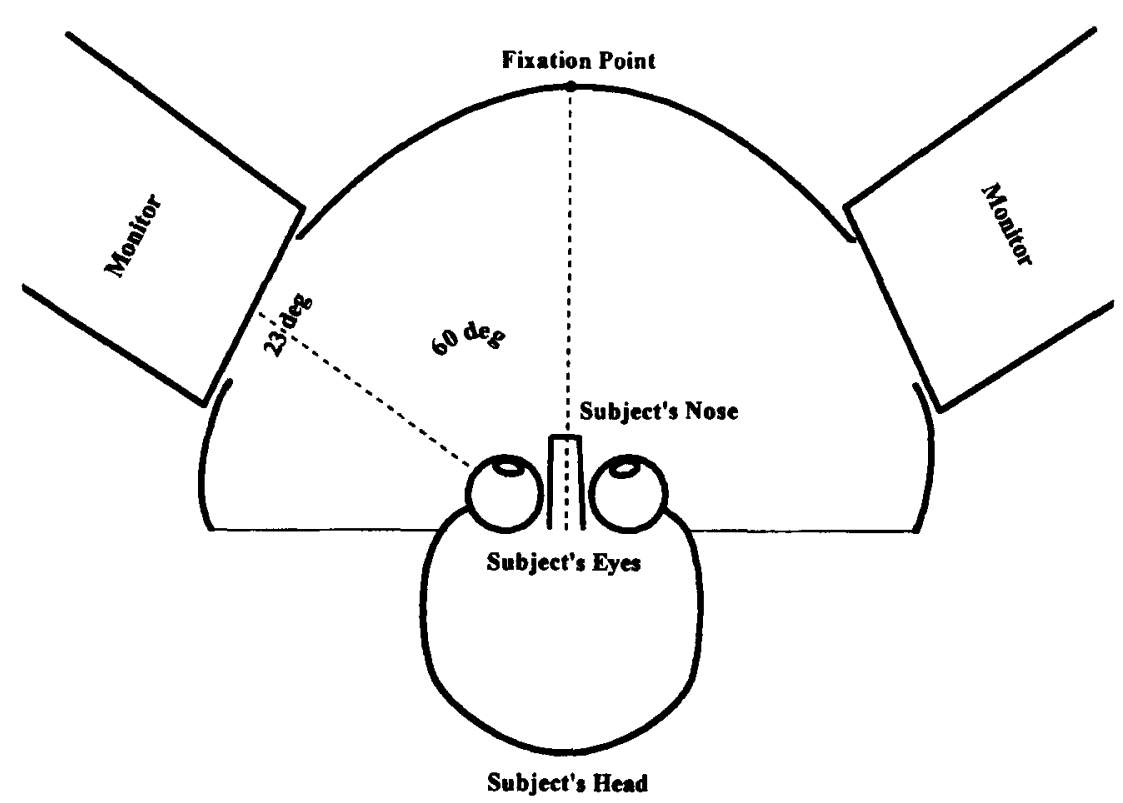

Figure 1. Bird's-eye view of the experimental setup. The size of the subject's head has been enlarged for the sake of clarity.

\section{Procedure}

Vection is not perceived at the onset of the visual stimulation. There is a latency before the onset of this perception, and the stabilization of the vection occurs only after a delay (Brandt et al., 1973; Howard, 1986; Sauvan \& Bonnet, 1993). Consequently, the method of limits, not a forced choice paradigm (Bonnet, 1986), has been used for measuring the thresholds of linear vection. But there are elements of the forced choice method in our paradigm because the subjects were asked in which direction they perceived linear vection.

There is a discrepancy between the subject's perception of vection and the knowledge of his/her motionlessness. This discrepancy is strengthened by the absence of any other sensorial information confirming the visual stimulation. Bonnet and de Schonen (1982) have shown that the proportion of trials in which a vection is perceived increases with the number of trials. Furthermore, with familiarity, the latency of the vection decreases down to a constant value. Consequently, we made a choice of working with subjects who had received practice (Bonnet, 1986). The number of subjects was therefore restricted.

The duration of each trial was $30 \mathrm{sec}$. The experimental conditions were randomized for each subject.

The subjects first received training in the situation before the experiment started. During the training, a series of stimuli was presented to each subject to verify whether he/she perceived rectilinear vection in each trial. The subjects reported whether or not they perceived self-motion and, when they did perceive it, they reported its direction. Within each $30-\mathrm{sec}$ trial, the direction of linear vection was constant. On the whole, Sauvan and Bonnet (1989) found that subjects perceived linear vection toward the slowest of the two motions - that is, the slowest apparent velocity.

At the end of each experimental session and during the training, subjects were asked whether they had already experienced induced self-motion when they were seated in an unmoving train and another train was moving in their peripheral visual field. If they answered affirmatively, they were asked whether the induced self-motion experience in the experiment or during the training was weaker or stronger than their previous real-life experience. The subjects were also asked whether this induced self-motion experience was weaker or stronger than their real-life experience of moving in a vehicle.

\section{EXPERIMENT 1}

The present experiment was designed to measure the thresholds of rectilinear vection induced by drifting sine wave gratings with respect to contrast $(C)$, temporal frequency (TF), spatial frequency (SF), and angular velocity $(V=\mathrm{TF} / \mathrm{SF})$.

\section{Method}

Four observers took part in Experiment 1. The spatiotemporal contrast sensitivity surface for rectilinear vection was measured with 3 subjects (M.B., J.-P.R., and X.S.). Contrast sensitivity was defined as the reciprocal of the contrast threshold. The spatial or temporal frequency and angular velocity thresholds were also measured with 3 subjects (M.B., N.B., and X.S.). Series of visual stimuli were used in which contrast, spatial frequency, temporal frequency, or angular velocity varied in such a way that the subject's perception of rectilinear vection decreased until it disappeared. The method used for measuring the thresholds was therefore the method of limits with descending series. This method was used because it had previously been observed that the thresholds measured with ascending series were at first larger than those measured with descending series and that the former then decreased until they became close to the latter (Sauvan, 1988). The contrast thresholds as a function of the spatial and temporal frequencies were measured for several pairs of spatial and temporal frequencies (Table 1).

The high spatial frequency thresholds were measured for the following temporal frequency values: $2.5,3.5,4.9,6.9$, and $9.6 \mathrm{~Hz}$. The contrast was equal to $60 \%$. For technical reasons, the low spatial frequency thresholds were not measured. The low and high temporal frequency thresholds were measured for the following spatial frequency values: $.23, .33, .48, .69$, and $.82 \mathrm{c} / \mathrm{deg}$. The contrast was equal to $60 \%$. The high and low angular velocity thresholds were 
Table 1

Pairs of Spatial and Temporal Frequencies $(\times)$ Used to Measure the Contrast Thresholds

\begin{tabular}{cccccc}
\hline & $.23 \mathrm{c} / \mathrm{deg}$ & $.32 \mathrm{c} / \mathrm{deg}$ & $.45 \mathrm{c} / \mathrm{deg}$ & $.63 \mathrm{c} / \mathrm{deg}$ & $.88 \mathrm{c} / \mathrm{deg}$ \\
\hline $2.5 \mathrm{~Hz}$ & $\times$ & & $\times$ & & \\
$3.5 \mathrm{~Hz}$ & & $\times$ & $\times$ & & \\
$4.9 \mathrm{~Hz}$ & $\times$ & $\times$ & $\times$ & $\times$ & $\times$ \\
$6.9 \mathrm{~Hz}$ & & & $\times$ & $\times$ & \\
$9.6 \mathrm{~Hz}$ & & & $\times$ & & $\times$ \\
\hline
\end{tabular}

measured by using a frequency matrix (TF/SF), and they were selected from one diagonal of that matrix. There were 30 experimental conditions.

\section{Results and Discussion}

All the thresholds are the means of 3 subjects' responses. The interindividual variability was estimated by means of the coefficient of variation $\mathrm{CV}=\left(S_{n-1} / M\right)^{*} 100$, $S_{n-1}$ being the sample standard deviation, and $M$ the mean. This coefficient did not vary systematically as a function of the experimental conditions. Its mean value was $26.2 \%$.

The spatiotemporal contrast sensitivity surface for rectilinear vection is shown in Figure 2. There is a zone of maximal contrast sensitivity for rectilinear vection cen- tered on low spatial and middle temporal frequencies. The best contrast sensitivity is located at about $0.40 \mathrm{c} / \mathrm{deg}$ and $6 \mathrm{~Hz}$.

As for the high spatial frequency thresholds, there is an optimum sensitivity for rectilinear vection of about $.80 \mathrm{c} / \mathrm{deg}$ at $4.9 \mathrm{~Hz}$. The ratio between the lower and higher thresholds was only 1.2. In the case of the high temporal frequency thresholds, there is an optimum sensitivity for rectilinear vection of about $14 \mathrm{~Hz}$ at $.48 \mathrm{c} / \mathrm{deg}$. The optimum sensitivity for low temporal thresholds is $1.67 \mathrm{~Hz}$ at $.48 \mathrm{c} / \mathrm{deg}$. The low velocity threshold is $4 \% \mathrm{sec}$ and the high velocity threshold is about $19^{\circ} / \mathrm{sec}$. According to the equation $V=\mathrm{TF} / \mathrm{SF}$ defined above, the former corresponds to a spatial frequency of $.83 \mathrm{c} / \mathrm{deg}$ and a temporal frequency of $3.3 \mathrm{~Hz}$, and the latter to a spatial frequency of $.38 \mathrm{c} / \mathrm{deg}$ and a temporal frequency of $7.32 \mathrm{~Hz}$. The results are coherent. Figure 3, constructed by plotting the values of the spatial frequency, temporal frequency, and velocity thresholds in terms of combinations of spatial and temporal frequencies, shows there is a spatiotemporal domain (or zone) of perception of rectilinear vection. The faster and slower velocities were $40.4^{\circ} / \mathrm{sec}$ and $3.48 \% \mathrm{sec}$, respectively. Moreover, the zone of maximal contrast sensitivity for rectilinear vection is inside the spatiotemporal domain of perception of rectilinear vection

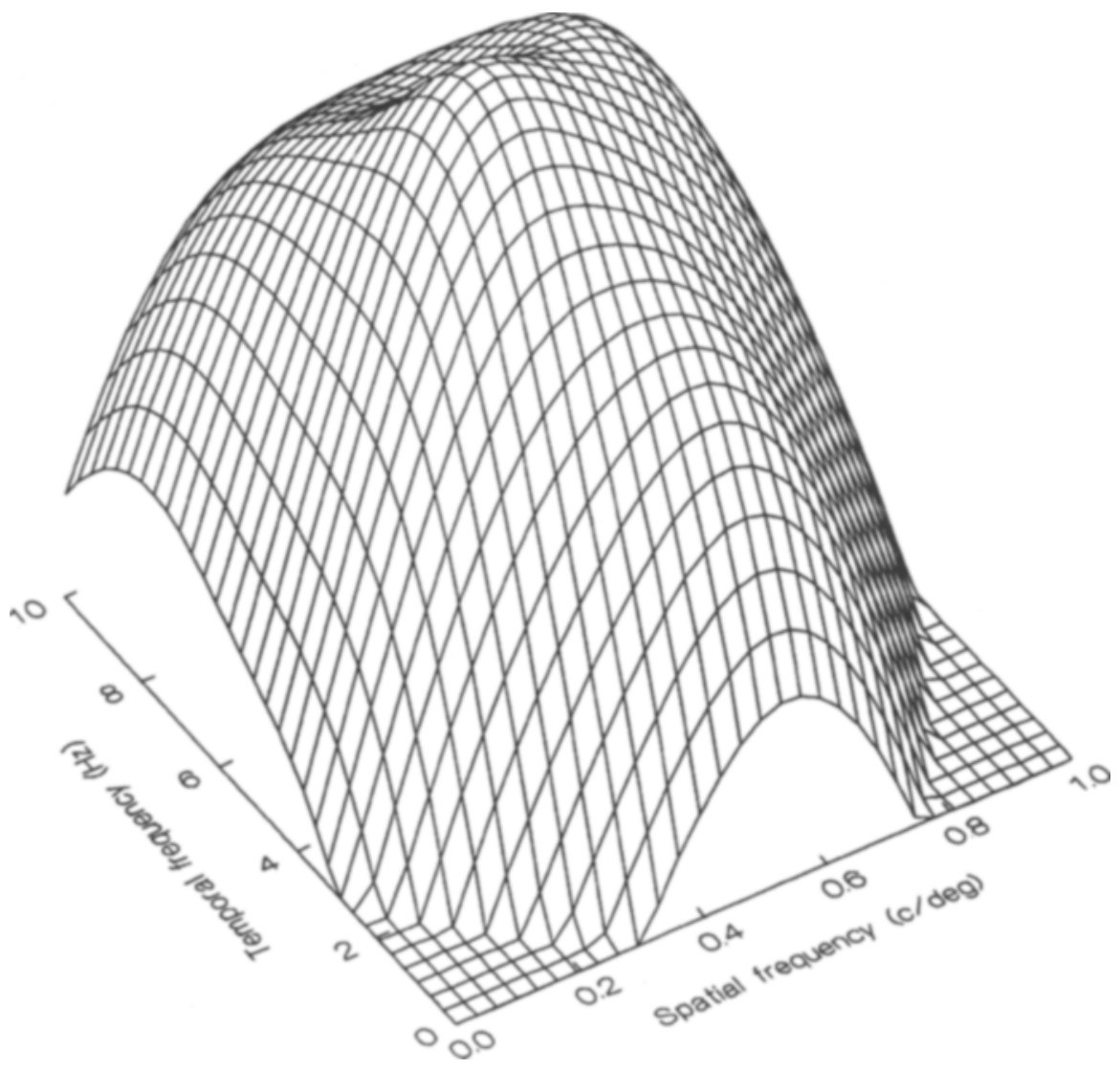

Figure 2. Spatiotemporal contrast sensitivity surface for rectilinear vection. The contrast sensitivity was plotted as a function of spatial frequency and temporal frequency. The distance-weighted least squares smoothing method was used to draw the surface. 


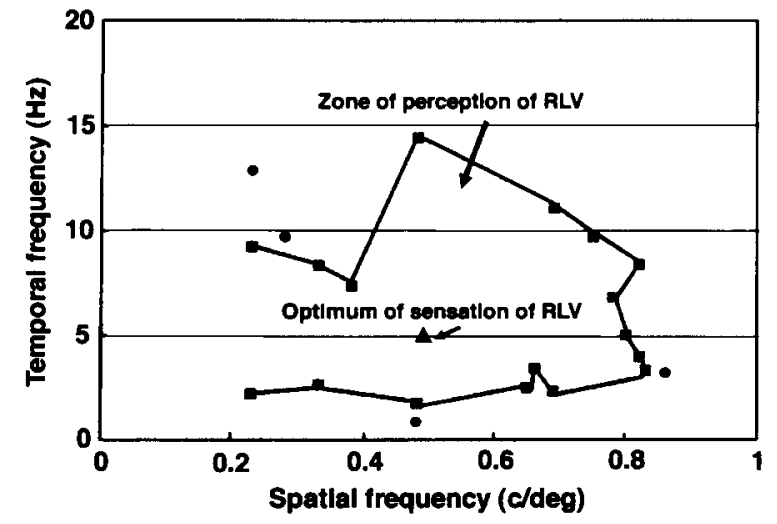

Figure 3. Spatiotemporal zone of perception of linear vection. Linear vection was not perceived outside this zone. Each pair of spatial and temporal frequencies (filled squares) corresponds to a threshold of perception of the rectilinear vection measured in Experiment 1. The spatial frequency, temporal frequency, and velocity thresholds were used to trace the figure. The thresholds to detect the movement of the drifting sine wave gratings (filled circles) measured in Experiment 2 are also shown.

(see Figures 2 and 3). Finally, the optimum of perception of linear vection which corresponds to an angular velocity of $10.2 \% \mathrm{sec}$ - that is, a spatial frequency of $0.49 \mathrm{c} / \mathrm{deg}$ and a temporal frequency of $5 \mathrm{~Hz}$ (Sauvan \& Bonnet, 1989)--is also inside this spatiotemporal domain (Figure 3).

\section{EXPERIMENT 2}

Experiment 2 was designed to measure thresholds for detecting the movement of drifting sine wave gratings and to compare them with the thresholds for perceiving rectilinear vection measured in Experiment 1.

\section{Method}

Three subjects (C.T., M.B., and X.S.) took part in Experiment 2. As in Experiment 1, the stimuli were designed by varying the temporal frequency or angular velocity until the subjects' perception of rectilinear vection disappeared. The method used for measuring the thresholds was also the method of limits with descending series.
There were four experimental conditions: low temporal frequency $\left(\mathrm{TF}_{l}\right)$, high temporal frequency $\left(\mathrm{TF}_{\mathrm{h}}\right)$, low angular velocity $\left(V_{1}\right)$, and high angular velocity $\left(V_{\mathrm{h}}\right)$. These conditions were as those in Experiment 1 . The contrast was $60 \%$ in all conditions. The experimental conditions were randomized for each subject. Thresholds for detecting the movement of drifting sine wave gratings were calculated by using $V=\mathrm{TF} / \mathrm{SF}$ (see Experiment 1).

\section{Results and Discussion}

The lowest threshold for detecting the movement of drifting sine wave gratings was $1.76^{\circ} / \mathrm{sec}$. The corresponding coefficient of interindividual variation was $16.2 \%$. The highest threshold for detecting the movement of drifting sine wave gratings was $56 \% \mathrm{sec}$. The corresponding coefficient of interindividual variation was $21.8 \%$. These four thresholds lie outside the spatiotemporal domain of perception of linear vection (Figure 3). Bonnet and Chaudagne (1979) found a similar result. This suggests the spatiotemporal domain of detection of object motion contains the spatiotemporal domain of perception of induced linear self-motion.

\section{EXPERIMENT 3}

The aim of Experiment 3 was to measure thresholds of curvilinear vection for contrast and angular velocity. The variables manipulated to measure these thresholds were contrast, spatial frequency, temporal frequency, and angular velocity. Thresholds of induced self-motion in a curved path were defined as the smallest differences for inducing this vection, because asymmetrical visual stimuli were used.

\section{Method}

Two subjects took part in Experiment 3 (M.B. and X.S.). The paradigm described in the General Method section was also used in Experiment 3, but with a series of asymmetrical visual stimuli. The asymmetry $D(X)$ of the stimuli was given by the difference between the value $X_{1}$ of a given variable $X$ on one of the two monitors and the value $X_{2}$ of the same variable on the other monitor $\left[D(X)=\left(X_{1}-\right.\right.$ $\left.X_{2}\right)$ ]. These asymmetrical visual stimuli are powerful in inducing curvilinear vection (Sauvan \& Bonnet, 1989, 1993). The asymmetry $D(X)$ for one variable decreased from one trial to another, so that subjects perceived a progressively weaker curvilinear vection until the threshold was reached. There were six experimental conditions,

Table 2

Values Corresponding to the Relative Differential Thresholds of the Curvilinear Vection

\begin{tabular}{lcccccc}
\hline & \multicolumn{7}{c}{ Condition } \\
\cline { 2 - 7 } & $\mathrm{SF}$ & $\mathrm{TF}_{1}$ & $\mathrm{TF}_{\mathrm{h}}$ & $V_{1}$ & $V_{\mathrm{h}}$ & $C$ \\
\hline$C(\%)$ & 60 & 60 & 60 & 60 & 60 & 60 \\
$\mathrm{SF}(\mathrm{c} / \mathrm{deg})$ & 0.23 & 0.48 & 0.48 & 0.54 & 0.54 & 0.48 \\
$\mathrm{TF}(\mathrm{Hz})$ & 4.3 & 7.45 & 3.6 & 5.0 & 5.0 & 5.18 \\
$V(\mathrm{deg} / \mathrm{sec})$ & 18.70 & 15.52 & 7.5 & 9.26 & 9.26 & 10.79 \\
$C(\%)$ & 60 & 60 & 60 & 60 & 60 & 51.3 \\
SF $(\mathrm{c} / \mathrm{deg})$ & 0.38 & 0.48 & 0.48 & 0.71 & 0.45 & 0.48 \\
$\mathrm{TF}(\mathrm{Hz})$ & 4.3 & 4.35 & 6.27 & 3.8 & 6.77 & 5.18 \\
$V(\mathrm{deg} / \mathrm{sec})$ & $\mathbf{1 1 . 3 2}$ & $\mathbf{9 . 0 6}$ & $\mathbf{1 3 . 0 6}$ & $\mathbf{5 . 3 5}$ & $\mathbf{1 5 . 0 5}$ & 10.79 \\
$W$ & $\mathbf{3 9 . 4 7}$ & $\mathbf{4 1 . 6 2}$ & $\mathbf{4 2 . 5 7}$ & $\mathbf{4 2 . 2 2}$ & $\mathbf{3 8 . 4 7}$ & \\
\hline
\end{tabular}

Note-SF, spatial frequency; $\mathrm{TF}_{1}$, low temporal frequency; $\mathrm{TF}_{\mathrm{h}}$, high temporal frequency; $V_{\mathrm{l}}$, low angular velocity; $V_{\mathrm{h}}$, high angular velocity; $C$, contrast. See text for further details 
each of which was presented to the subjects twice. In five conditions, the thresholds were expressed as angular velocity thresholds (spatial frequency, SF; low temporal frequency, $\mathrm{TF}_{1}$; high temporal frequency, $\mathrm{TF}_{\mathrm{h}}$; low angular velocity, $V_{l}$; and high angular velocity, $V_{\mathrm{h}}$. In the contrast $(C)$ condition, the contrast threshold for curvilinear vection was measured. The values which were constant from one trial to another, as for each experimental condition, are shown in the upper part of Table 2.

\section{Results and Discussion}

The values which correspond to the measured angular velocity and contrast thresholds are shown in bold in the lower part of Table 2, along with the corresponding values of the other variables. There is a Weber's law such that a mean relative differential threshold of the angular velocities of the moving images equal to about $41 \%$ is necessary for inducing curvilinear vection. This ratio is defined as follows:

$$
W=\left[\left(V_{\mathrm{h}}-V_{1}\right) / V_{\mathrm{h}}\right] * 100,
$$

where $V_{\mathrm{I}}$ and $V_{\mathrm{h}}$ are the lowest and the highest velocities, respectively. The ratios $(W)$ are almost the same for the five experimental conditions (Table 2).

From the contrast $(C)$ condition, it appears that a contrast difference of about $9 \%$ is sufficient to induce curvilinear vection (Table 2). This result is understandable, because the apparent velocity of a moving stimulation is decreasing when its contrast is diminishing (Thompson, 1982). This happens when the temporal frequency of the stimulation is less than about $8 \mathrm{~Hz}$. This was the case in the present experiment. Consequently, an apparent velocity difference that is high enough induces curvilinear vection.

\section{GENERAL DISCUSSION}

A stationary central visual stimulation seems to affect the perception of a peripherally induced self-motion. Indeed, we observed that the removal of the difference between the mean luminance of the drifting sine wave gratings and that of the spatially homogeneous visual surroundings induces an inhibition of the perception of linear vection (Sauvan, 1988). This may be related to the increase of the the perceived structure of the visual space when the difference of luminance was removed. Further investigation is needed to study this phenomenon more precisely.

It was shown that linear vection can be perceived by using two low spatial frequency $(<1.0 \mathrm{c} / \mathrm{deg})$ stimulations of only $23^{\circ}$ of visual angle located at $60^{\circ}$ of eccentricity. Properties of the systems involved in the visual control of self-motion can be inferred from the spatial frequency range found in the present studies. In particular, the finding that low spatial frequency stimuli induce linear vection may be related to the finding that the receptive field width of the human motion detector units inferred from spatial frequency masking varies with respect to spatial frequency from $5.8^{\circ}$ at $.03 \mathrm{c} / \mathrm{deg}$ to $.05^{\circ}$ at $10 \mathrm{c} / \mathrm{deg}$ (Anderson \& Burr, 1989). This is similar to the receptive field width obtained with a summation technique (Anderson \& Burr, 1987). The motion-detector receptive fields decrease in size (in degrees) with increasing spatial fre- quency and have a length:width ratio of 1 , but the motionindependent units have elongated receptive fields with a length:width ratio of about 1.88 (Anderson, Burr, \& Morrone, 1991). Consequently, the receptive field size of the motion detector units that are stimulated seems to be essential for perceiving linear vection in the same way as are the size of the stimulus, its eccentricity, and the optical flow structure (Sauvan \& Bonnet, 1993).

According to Andersen and Braunstein (1985), two systems control ambient vision: a lower level system that would require peripheral vision, and a higher level system that would work in the central visual field and would process complex visual information such as depth cues. The more primitive ambient system has also been described as complementary to the focal system: The former would be involved in spatial orientation and localization; the latter, in object recognition (Wolpert, 1990). The primary characteristic of the more primitive system seems to be its sensitivity to low spatial frequencies. In the present experimental work, the spatiotemporal contrast sensitivity surface and the spatiotemporal domain of perception of peripheral rectilinear vection are centered on the low spatial and the middle temporal frequencies. In the same way, the values that correspond to the measured relative differential thresholds of peripheral curvilinear vection are low spatial and middle temporal frequencies. Consequently, the more primitive ambient system should be involved in the perception of linear vection. But it has been shown that the direction of peripherally induced curvilinear selfmotion is not always unambiguously perceived (Sauvan \& Bonnet, 1989), and the perceived velocity and degree of curvature of peripheral curvilinear vection vary differently (Sauvan \& Bonnet, 1993). Consequently, the more primitive ambient system is not sufficient for computing the curvilinear component of induced self-motion in a curved path accurately. This means that the higher level ambient system should also be involved in perceiving linear self-motion. Similarly, Frignon and Delorme (1992) have suggested that perceived self-motion triggered at low sinusoidal motion frequencies and at high sinusoidal motion amplitudes and frequencies could be under the control of the more primitive and the higher level ambient systems, respectively.

In short, a better understanding of the visual control of complex self-motions may be provided by the study of the properties of complex induced self-motions if the latter are investigated in different situations in which various central and/or peripheral visual stimuli are manipulated.

\section{REFERENCES}

Andersen, G. J. (1986). Perception of self-motion: Psychophysical and computational approaches. Psychological Bulletin, 99, 52-65.

ANdersen, G. J., \& BraunsteIn, M. L. (1985). Induced self-motion in central vision. Journal of Experimental Psychology: Human Perception \& Performance, 11, 122-132.

ANdERSEN, G. J., \& DYre, B. P. (1989). Spatial orientation from optic flow in the central visual field. Perception \& Psychophysics, 45, 453-458.

ANDERSON, S. J., \& BURR, D. C. (1987). Receptive field size of human motion detection units. Vision Research, 27, 621-635.

ANDERson, S. J., \& BURR, D. C. (1989). Receptive field properties of 
human motion detector units inferred from spatial frequency masking. Vision Research, 29, 1343-1358.

Anderson, S. J., Burr, D. C., \& Morrone, M. C. (1991). Twodimensional spatial and spatial-frequency selectivity of motionsensitive mechanisms in human vision. Journal of the Optical Society of America $A, \mathbf{8}, 1340-1351$.

Berthoz, A., \& Droulez, J. (1982). Linear self-motion perception. In A. H. Wertheim, W. A. Wagenaar, \& H. W. Leibowitz (Eds.), Tutorials in motion perception (pp. 157-199). London: Plenum.

Berthoz, A., Pavard, B., \& Young, L. R. (1975). Perception of linear horizontal self-motion induced by peripheral vision (linear vection): Basic characteristics and visual-vestibular interactions. Experimental Brain Research, 23, 471-489.

BONNET, C. (1986). Manuel pratique de psychophysique [Textbook of psychophysics]. Paris: Armand Colin.

Bonnet, C., \& Chaudagne, N. (1979). Comparison of contrast thresholds for object-motion and for self-motion in different directions. $E x$ perimental Brain Research, 36, R6.

BONNET, C., \& DE SCHONEN, S. (1982). Étude du contrôle visuel de la stabilisation posturale et de son développement avec l'âge [Study of the visual control of postural stabilization and its development with age]. (Unpublished INSERM contract final report). Paris: Université René Descartes, Laboratory of Experimental Psychology.

Brandt, T., DichGans, J., \& Koenig, E. (1973). Differential effects of central versus peripheral vision on egocentric and exocentric motion perception. Experimental Brain Research, 16, 476-491.

BütTNER, U., \& BuetTNER, U. V. (1978). Parietal cortex (2v) neuronal activity in the alert monkey during vestibular and optokinetic stimulation. Brain Research, 153, 392-397.

BÜTTNER, U., \& HENN, V. (1976). Thalamic unit activity in the alert monkey during natural vestibular stimulation. Brain Research, 103, 127-132.

BütTner, U., \& Henn, V. (1981). Circular vection: Psychophysics and single-unit recordings in the monkey. In B. Cohen (Ed.), Vestibular and oculomotor physiology: International meeting of the Barany Society. (Annals of the New York Academy of Sciences, Vol. 374, pp. 274-283). New York: New York Academy of Sciences.

BÜTTNER, U., \& LANG, W. (1979). The vestibulocortical pathway: Neurophysiological and anatomical studies in the monkey. Progress in Brain Research, 50, 581-588.

DiChGaNS, J., \& BRANDT, T. (1978). Visual-vestibular interaction: Effects on self-motion perception and postural control. In R. Held, H. W. Leibowitz, \& H. L. Teuber (Eds.), Handbook of sensory physiology (Vol. 8, pp. 755-804). Berlin: Springer-Verlag.

Frignon, J.-Y., \& Delorme, A. (1992). Roll, pitch, longitudinal, and yaw vection visually induced by optical flow in flight simulation conditions. Perceptual \& Motor Skills, 74, 935-955.

GiBson, J. J. (1979). The ecological approach to perception. Boston: Houghton Mifflin.

HowARD, I. P. (1986). The perception of posture, self-motion and visual vertical. In K. R. Boff, L. Kaufman, \& J. P. Thomas (Eds.), Handbook of perception and human performance (Vol. 1, pp. 18-1 to 18-62). New York: Wiley.

LAPPE, M., \& RAUSCHECKER, J. P. (1993). A neural network for the processing of optic flow from ego-motion in man and higher mammals. Neural Computation, 5, 374-391.

Leibowitz, H. W., Rodemer, C. S., \& Dichgans, J. (1979). The independence of dynamic spatial orientation from luminance and refractive error. Perception \& Psychophysics, 25, 75-79.

Lestienne, F., Soechting, J., \& Berthoz, A. (1977). Postural readjustments induced by linear motion of visual scenes. Experimental Brain Research, 28, 363-384.

Post, R. B. (1988). Circular vection is independent of stimulus eccentricity. Perception, 17, 737-744.

Probst, T., Straube, A., \& Bles, W. (1985). Differential effects of ambivalent visual-vestibular-somatosensory stimulation on the perception of self-motion. Behavioural Brain Research, 16, 71-79.

Rauschecker, J. P., von Grünau, M. W., \& Poulin, C. (1987). Centrifugal organization of direction preferences in the cat's lateral suprasylvian visual cortex and its relation to flow field processing. Journal of Neuroscience, 7, 943-958.
SaUvan, X. M. (1988). Étude psychophysique de la vection curvilinéaire et de la vection rectilinéaire [Psychophysical study of curvilinear vection and rectilinear vection]. Unpublished doctoral dissertation, Université Pierre et Marie Curie, Paris.

SaUVAN, X. M., \& Bonnet, C. (1988). Thresholds and variations of the forward rectilinear and curvilinear vections in man. In Proceedings of the EBBS workshop "Visual processing of form and motion," University of Tübingen, Germany.

Sauvan, X. M., \& Bonnet, C. (1989). Les sensations de déplacement curvilinéaire générées visuellement [Perception of visually induced curvilinear self-motion]. Psychologie Française, 34, 19-24.

SaUvan, X. M., \& BonNET, C. (1993). Properties of curvilinear vection. Perception \& Psychophysics, 53, 429-435.

Sauvan, X. M., \& Peterhans, E. (1995). Neural integration of visual information and direction of gravity in prestriate cortex of the alert monkey. In T. Mergner \& F. Hlavacka (Eds.), Multisensory control of posture (pp. 43-49). New York: Plenum.

Stoffregen, T. A. (1985). Flow structure versus retinal location in the optical control of stance. Journal of Experimental Psychology: Human Perception \& Performance, 11, 554-565.

Stoffregen, T. A., Schmuckler, M. A., \& Gibson, E. J. (1987). Use of central and peripheral optical flow in stance and locomotion in young walkers. Perception, 16, 113-119.

TANAKA, K., \& SaITO, H. (1989). Analysis of motion of the visual field by direction, expansion/contraction, and rotation cells clustered in the dorsal part of the medial superior temporal area of the macaque monkey. Journal of Neurophysiology, 62, 626-641.

TELFORD, L., \& Frost, B. J. (1993). Factors affecting the onset and magnitude of linear vection. Perception \& Psychophysics, 53, 682-692.

Telford, L., Spratley, J., \& Frost, B. J. (1992). Linear vection in the central visual field facilitated by kinetic depth cues. Perception, 21, 337-349.

Thompson, P. (1982). Perceived rate of movement depends on contrast. Vision Research, 22, 377-380.

VANNI-MERCIER, G., \& MAGNIN, M. (1982). Single neuron activity related to natural vestibular stimulation in the cat's visual cortex. $E x$ perimental Brain Research, 45, 451-455.

WAESPE, W., \& HENN, V. (1977). Neuronal activity in the vestibular nuclei of the alert monkey during vestibular and optokinetic stimulation. Experimental Brain Research, 27, 533-538.

WAESPE, W., \& HENN, V. (1978). Conflicting visual-vestibular stimulation and vestibular nucleus activity in alert monkeys. Experimental Brain Research, 33, 203-211.

WOLPERT, L. (1990). Field-of-view information for self-motion perception. In R. Warren \& A. H. Wertheim (Eds.), Perception and control of self-motion (pp. 101-126). Hillsdale, NJ: Erlbaum.

WURTZ, R. H., \& DUFFY, C. J. (1992). Neuronal correlates of optic flow stimulation. In. B. Cohen, D. L. Tomko, \& F. Guedry (Eds.), Sensing and controlling motion: Vestibular and sensorimotor function (Annals of the New York Academy of Sciences, Vol. 656, pp. 205-219). New York: New York Academy of Sciences.

\section{NOTES}

1. Optical flow is defined as lawful transformations of the optic array generated by an observer's ego motion in a stable environment (Gibson, 1979).

2. Under everyday circumstances, optical flow associated with selfmotion in the sagittal plane has radial structure in the central visual field and lamellar structure in the peripheral visual field (see Andersen, 1986).

3 . This is the perception of moving backward or forward in the sagittal plane triggered by a central visual stimulation.

4. This is the induced perception of moving leftward or rightward in the frontoparallel plane.

5. These displays simulated self-motion in the sagittal plane.

6 . These displays simulated self-motion relative to the frontoparallel plane.

(Manuscript received September 1, 1993; revision accepted for publication March 4, 1995.) 